\title{
ECC
}

\section{Hyaluronic acid hydrogel nanoscaffolds: production and assessment of the physicochemical properties}

\author{
Elham Ahmadiana, Solmaz Maleki Dizaja, Simin Sharifia, Aziz Eftekharib, \\ Mohammad Samieic,* \\ aDental and Periodontal Research Center, Tabriz University of Medical Sciences, Tabriz, \\ 5165665931 Iran
}

${ }^{b}$ Pharmacology and Toxicology Department, Maragheh University of Medical Sciences, Maragheh,5516773111 Iran

cFaculty of Dentistry, Tabriz University of Medical Sciences, Tabriz, 5165665931 Iran

Received: 13 October 2019, Accepted: 02 November 2019, Published: 23 November 2019

\begin{abstract}
Hyaluronic acid (HA) is the major constituent of the extracellular matrix (ECM) and mainly acts as a filler in the connective tissues. The goal of the current study was to develop and evaluate the physiochemical properties of HA hydrogel nanoscaffolds. Chemical precipitation technique and the use of glutaraldehyde-based crosslinking were utilized to prepare the nanoscaffolds. Dynamic light scattering (DLS), zeta sizer (measurement of zeta potential), scanning electron microscopy (SEM), and fourier transform infrared spectroscopy (FTIR) were performed to characterize the produced HA hydrogel nanoscaffolds. A relatively bimodal and monodispersed HA nanohydrogels were obtained and the mean particle size was reported to be 291.30 $\mathrm{nm}$. In addition, the results showed that zeta potential had a negative value (-5.96 mv). The FTIR results proved the crosslinking of the constructed scaffold. The observed physiochemical specifications proposed that HA hydrogel nanoscaffolds could hold promise in different biomedical implementations, in particular, tissue regeneration.
\end{abstract}

Keywords: Hyaluronic acid; hydrogel; nanoscafold; characterization.

\section{Introduction}

Different covalent or non-covalent crosslinks in the three-dimensional structure of hydrogels enables them to absorb bulky volumes of water [1-3]. When a chemical crosslinking is applied, the stability of HA structures is augmented [4,5]. Regarding HA, hydrogels are usually fabricated through chemical alterations in the solution via different organic and/or toxic reagents [6].

Hyaluronic acid (HA) as the potential candidate in construction of the artificial matrices, shows several superiorities in tissue engineering [7]. It is a biodegradable, biocompatible and bioactive compound which does not generate immunological reactions [8].

Application of HA has offered different advantages in the realm of

*Corresponding author: Mohammad Samiei

Tel: +98 (41) 33355965, Fax: +98 (41) 33355965

E-mail: samiei.moh@gmail.com

Eurasian Chem. Commun., (2020) 51-58

Page 51 
biomedicine. As a prominent factor in mediation of different cellular functions, one line of thought has been its application in the clinic [9]. Transformation of HA into the bendable sheets during the formation of hydrogels has been reported in preclinical and clinical observations [10]. Different cells or therapeutic compounds could be delivered into the target tissues via HA-based hydrogels. Moreover, these structures can influence the biological function of the cells and alter their behavior. For instance, the differentiation of stem cells to a distinct tissue could be modified by the interaction of HA hydrogels with cellular signaling [11]. This effect is not observed using polymer-based materials [6]. Future research will witness the production of novel biomaterials with exceptional and remarkable properties.

HA-based constructs provide safe and anti-microbial surfaces in the fabricated biomaterials, which could be applied in trauma, orthopedics and dental surgery [12]. It has been observed that topical HA has advantageous effect in tackling dental problems such as periodontitis and gingivitis [13]. Also, HA not only enhances the therapeutical effects of delivered drugs in different diseases but also plummets their adverse effects [14]. Hydrogel nanoscaffolds have been utilized in creation of prevasculrized dental pulp-like structures and cartilage tissue for dentin and cartilage regeneration $[15,16]$.

The unique structure of HA hydrogels allows them increase cell attachment, growth and speeding in the field of stem cell therapy. Injectable hydrogels are suitable candidates of scaffolds in several tissue engineering processes including pulp regeneration [17].
Nanotechnology has gained increasing momentum in fabrication of cost-effective nanosystems by different strategies. Nanohydrogels benefit from the advantages of both hydrogels and the nanosized systems. Nanohydrogels have grabbed great attention since that they can act as unique controlled drug delivery systems, biological sensors, absorbents and biomimetic structures [18]. Nanohydrol can be developed using either natural or synthetic polymers for the abovementioned applications [19].

The use of HA in this context can significantly enhance the viscoelastic specification of nanohydrogels and affect the conservation of the volumes and shapes in the aqueous environments in an upward trend. Ionical, chemical or physical crosslinkings could be used in their fabrication. Similar to hydrogels, nanohydrogels are biocompatible and biomimetic materials due to their hydrophilicity and softness properties [20]. For instance, nanohydrogels have been extensively utilized in the synthesis of artificial extracellular matrix (ECM) to assess the cell functions such as proliferation and differentiation under different circumstances [21,22].

The objective of the current investigation was to produce and evaluate physicochemical properties of HA hydrogel nanoscaffolds.

\section{Experimental}

General

Sodium hyaluronate and dimethyl sulfoxide (DMSO) were obtained from Sigma-Aldrich Co. Glutaraldehyde was purchased from Merck. All other chemicals were analytical grade and utilized without additional purification.

Synthesis of HA hydrogel nanoscaffolds 
HA hydrogel nanoscaffolds were synthetized using solvent/non-solvent chemical precipitation method. In brief, an equal volume of DMSO was added to a $0.5 \% \mathrm{w} / \mathrm{v}$ HA sodium salt solution in bidistilled water in a dropwised manner $(1 \mathrm{~mL} / \mathrm{min})$ under magnetic stirring $(500 \mathrm{rpm})$. Then, glutaraldehyde (200 microliter) was added to the reaction mixture to produce crosslinking. The $\mathrm{pH}$ set to 4.5 using $\mathrm{HCl}(0.1 \mathrm{M})$ and the reaction continued for $4 \mathrm{~h}$ under magnetic stirring $(500 \mathrm{rpm})$. The prepared hydrogel was washed in ethanol and water to remove the extra solvents and reagents. The produced hydrogel was then dried by lyophilization process.

\section{Characterization of the nanoscaffold}

Particle size and morphology

Dynamic light scattering (DLS) method was used to determine the average diameter of the nanoscafflod technique (Malvern, United Kingdom). Moreover, the morphology of the prepared nanoscaffold was analyzed via scanning electron microscope (SEM) analysis (SEM, TESCAN, USA). Increments of water/ethanol were utilized to dehydrate every porous nanohydrogels in a serial manner for $10 \mathrm{~min}$. The nanoscaffold was left in ethanol $(100 \%)$ overnight followed by air drying and vacuuming before imaging. Finally, a thin layer of gold was coated on the gels prior to the imaging.

\section{Zeta potential measurements}

Zeta sizer (Malvern, United Kingdom) was applied to determine the zeta potential of the HA hydrogel nanoscaffold at $25 \pm 1^{\circ} \mathrm{C}$. Zeta potential assesses the surface charge of the materials. Distilled water was used to dilute the freshly prepared nanoscaffold and afterward it was placed in the capillary cell of the sizer.
Fourier transform infrared spectroscopy (FTIR) analysis

The chemical structure of the HA hydrogel nanoscaffolds was assessed via FTIR analysis. A FTIR spectrophotometer gave the FTIR patterns using the $\mathrm{KBr}$ disk technique from 400 to $4000 \mathrm{~cm}-1$ at a resolution of $4 \mathrm{~cm}-1$.

\section{Results and discussion}

Particle size and morphology

Chemical precipitation crosslinking approach is a common method in preparation of HA hydrogels [23]. The growth and nucleation of the material are highly related to the chemical reaction rate, which modulates particle size and size distribution [24]. Establishment of proficient crosslinking agents can regulate the particle size and size distribution as well. Particle size distributions of the developed nanoscaffolds are illustrated in Figure 1. The mean diameter of the HA hydrogel-based nanoscaffold was reported to be $291.3 \mathrm{~nm}$. Moreover, the Polydispersity index of 0.34 was recorded showing a bimodal monodispersed size distribution in our experiment. This also shows a narrow size distribution. Sahiner and Jia reported the preparation of one-step synthesis of HA microhydrogel. They prepared uniform spherical microparticles (4.88 $\mu \mathrm{m}$ in size). The authors believed that for the optimal HA hydrogel particle preparation, the chosen preparation method and the MW of HA are so important parameters. The prepared microhydrogeles by them showed the broad size distribution. Therefore, they used filtration paper to obtain the narrow size distribution. They also used divinyl sulfone as crosslinker. According to their discussion, the alkaline condition needing for covalent crosslinking of HA with divinyl sulfone 
leads to the significant decrease in stable particles [25]. solution viscosity and then forming of

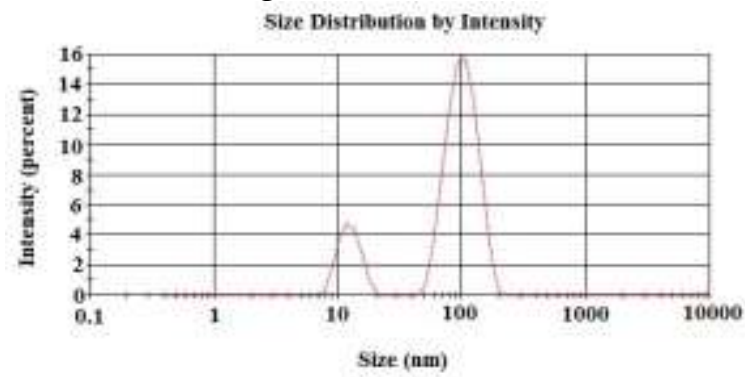

Figure 1. The size distribution of HA hydrogel nanoscaffold

The nano-pores and gel structure of the nanoscaffold was assessed via SEM (Figure 2). The construction method and type of the hydrophobic substituents are the main factors, which affect the morphological structure of a hydrogel system [26].

In a work by Collins and Birkinshaw, the prepared HA hydrogel showed an average pore size ranging from 167 to $215 \mu \mathrm{m}$. They believed that the prepared hydrogel would be an appropriate place for cell proliferation [27]. It was shown here that the pores of the prepared nanoscaffold were in network form which resembles the ECM. Also, SEM results indicated that the pore sizes were about $100 \mathrm{~nm}$. However, the precise pore size could not be measured from these SEM images.

Bulk gels or the traditional HA hydrogels exhibit macroscopic network, which contains indiscriminate interconnected HA chains [28]. This structure is missing the functional diversity of the endogenous ECM [29]. The formation of crosslinking reaction in the microscopic reaction vessel results in the production of $\mathrm{HA}$ hydrogel particles [6]. Tunable size, ample internal space and large surface area and the presence of functional group are the main advantages of the HA hydrogel particles [30]. Furthermore, sequestration of therapeutically active components which control the liberation of HA hydrogel particles and trigger their biological activity occurs following an accurate synthesis [6]. Microscopic and nano-scale synthesis methods as technological drivers of innovation as well as the use of well-established crosslinking agents have introduced novel surrogates for traditional HA hydrogels. Other structures have also been developed in the recent years. It has been shown that nanofibrous HA hydrogels have anisotropic specification and substitute cell-matrix interactions [6]. 


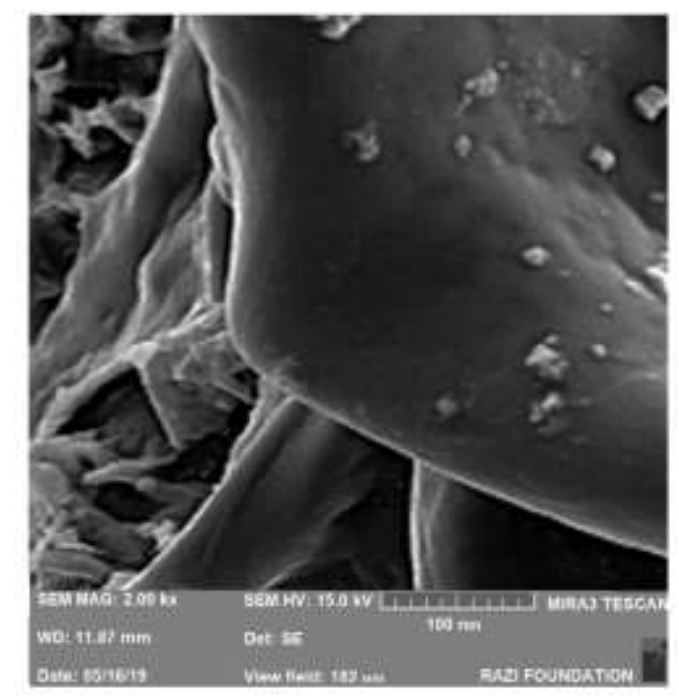

Figure 2. The scanning electron microscopy of HA hydrogel nanoscaffold

\section{Zeta potential measurements}

The zeta potential of the prepared HA hydrogel nanoscaffold has been presented in Figure 3. A spontaneous surface electrical charge can be obtained in every material after exposure to a polar environment such as water [31]. Literature search fetches some mechanisms in this context such as ionization of surface groups, the differences in the electron affinity of 2 phases, differential ion dissolution, differential ion adsorption, isomorphism replacement, and surface anisotropy. Among them, the surface group ionization is the chief mechanism regarding all surfaces that are metal oxide or have amino and/or carboxyl groups such as polyelectrolytes, ionic polymers and proteins [32,33].

The suspension stability is also anticipated via zeta potential. The best stability is commonly defined in zeta potentials above $\pm 60 \mathrm{mV}$, whereas particle aggregation occurs in zeta potential less than \pm 5 . Acceptable short-term stability is obtained in the zeta potentials between these two ranges $[34,35]$. Surface charge of the materials is a determinant factor in their biological function. For instance, the response of the body to the scaffolds utilized in bone regeneration is in a tight connection with the surface specification of the materials. It has been shown that binding and growth of osteoblasts is higher in negatively charged materials in comparison to the positive surfaces. The incorporation of a drug within or adsorption on the surface of a material is also affected by zeta potential [36,37].

For the prepared HA hydrogel by Sahiner and Jia, the amount of zeta potential was obtained $-40 \mathrm{mV}$, representing the high stability of the particles suspention as well as the protection of the carboxylic acid functionality on HA after particle formation. The authors believed that due to the effective removing process of the utilized surfactant, the observed negative charge is unlikely to be related to surfactants [25].

Sakulwech et al. have demonstrated that the increment of HA content leads to the generation of larger nanoparticles with lower zeta potential since HA has a negative charge [38]. 


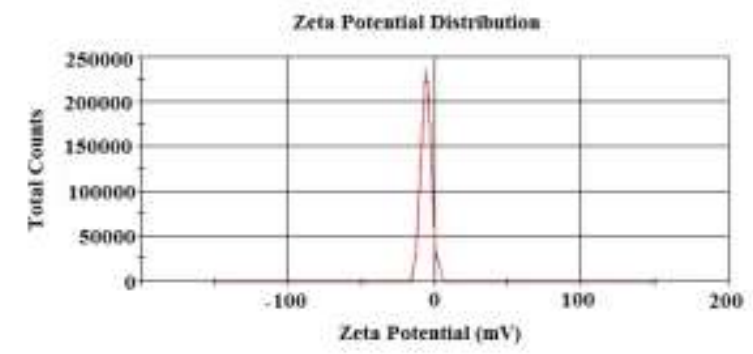

Figure 3. The zeta potential of HA hydrogel nanoscaffold

\section{FTIR analysis}

The sort of bindings generated during the modification of $\mathrm{HA}$ is determined via FTIR method (Figure 4), which characterizes cross-linked or linear HA $[39,40]$. In a work conducted by Alsibani et al., the presence of alkyl chain in the crosslinking agent has been observed in cross-linked HA (bands were seen between 2850 and $2930 \mathrm{~cm}^{-}$ $\left.{ }^{1}\right)$. In addition, when the amount of the cross-linker is enhanced, the band intensity of the carboxyl group declines [40]. However, in another study, no significant differences were observed between the spectra of cross-linked and linear HA excluding a band at $1650 \mathrm{~cm}^{-}$ 1 [41]. The results in our study indicated the proper crosslinking of the HA hydrogel nanoscaffolds.

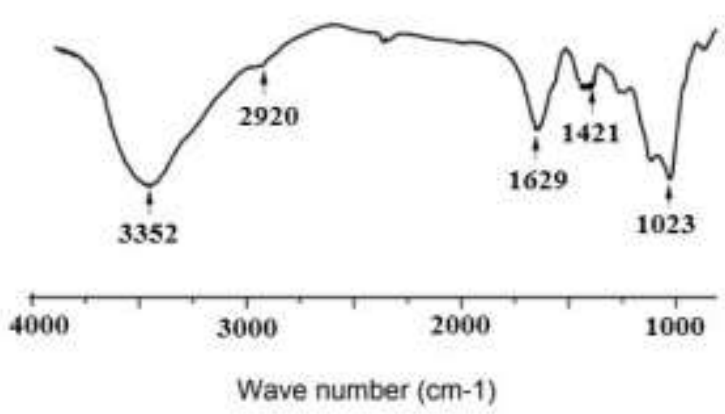

Figure 4. Fourier transform infrared spectroscopy (FTIR) of HA hydrogel nanoscaffold

\section{Conclusion}

The purpose of this experiment was to prepare and evaluate the physicochemical properties of HA hydrogel nanoscaffolds which was developed using chemical precipitation technique and glutaraldehyde was utilized as a crosslinking chemical. The produced HA hydrogel nanoscaffolds were then characterized by DLS, SEM, zeta sizer and FTIR. The results displayed relatively monodisperse and biomodal HA hydrogel nanoscaffolds with the mean particle size of 291.30 $\mathrm{nm}$ with the negative values of the zeta potential $(-5.96 \mathrm{mv})$. These results propose that HA hydrogel nanoscaffolds are potential candidates in the field of stem cell therapy and tissue regeneration. The unique properties of the $\mathrm{HA}$ as a natural component of ECM and the benefits of nanotechnology in fabrication of hydrogels introduces a novel alternative system to the existing HA bulk gels.

\section{Conflict of interest}

None.

\section{Acknowledgments}

This is a report of a database from a Postdoc research entitled "Synthesis and characterization of hyaluronic acid hydrogel nano-scaffolds and their role in odontoblastic differentiation of 
dental pulp stem cells" registered and funded by a grant (Grant number: 62476) from Vice Chancellor for Research (VCR) of Tabriz University of Medical Sciences (TUOMS), Tabriz, Iran.

\section{References}

[1] A.S. Hoffman, Adv. Drug Deliv. Rev., 2012, 64, 18-23.

[2] E. Caló, V.V. Khutoryanskiy, Eur Polym J, 2015, 65, 252-267.

[3] T.R. Hoare, D.S. Kohane, Polymer, 2008, 49, 1993-2007.

[4] T. Segura, B.C. Anderson, P.H. Chung, R.E. Webber, K.R. Shull, L.D. Shea, Biomaterials, 2005, 26, 359-371.

[5] G. Tripodo, A. Trapani, M.L. Torre, G. Giammona, G. Trapani, D. Mandracchia, Eur J Pharm Biopharm, 2015, 97, 400-416.

[6] X. Xu, A.K. Jha, D.A. Harrington, M.C. Farach-Carson, X. Jia, Soft Matter, 2012, 8, 3280-3294.

[7] J. Baier Leach, K.A. Bivens, C.W. Patrick Jr, C.E. Schmidt, Biotechnol Bioeng, 2003, 82, 578-589.

[8] H. Tan, C.R. Chu, K.A. Payne, K.G. Marra, Biomaterials 2009, 30, 24992506.

[9] J.A. Burdick, G.D. Prestwich, $A d v$ Mater, 2011, 23, H41-H56.

[10] K.T. Dicker, L.A. Gurski, S. Pradhan-Bhatt, R.L. Witt, M.C. FarachCarson, X. Jia, Acta biomaterialia, 2014, 10, 1558-1570.

[11] S. Gerecht, J.A. Burdick, L.S. Ferreira, S.A. Townsend, R. Langer, G. Vunjak-Novakovic, PNAS, 2007, 104, 11298-11303.

[12] C. Romanò, E. De Vecchi, M. Bortolin, I. Morelli, L. Drago, J Bone Jt Infect, 2017, 2, 63.

[13] M. Casale, A. Moffa, P. Vella, L. Sabatino, F. Capuano, B. Salvinelli, M.A. Lopez, F. Carinci, F. Salvinelli, SAGE Publications Sage UK: London, England, 2016.
[14] N.M. Salwowska, K.A. Bebenek, D.A. Żądło, D.L. Wcisło-Dziadecka, $J$ Cosmet Dermatol, 2016, 15, 520-526.

[15] A. Athirasala, F. Lins, A. Tahayeri, M. Hinds, A.J. Smith, C. Sedgley, J. Ferracane, L.E. Bertassoni, Scientific reports, 2017, 7, 3323.

[16] R. Levato, W.R. Webb, I.A. Otto, A. Mensinga, Y. Zhang, M. van Rijen, R. van Weeren, I.M. Khan, J. Malda, Acta biomaterialia, 2017, 61, 41-53.

[17] Y. Inuyama, C. Kitamura, T. Nishihara, T. Morotomi, M. Nagayoshi, Y. Tabata, K. Matsuo, K.K. Chen, M. Terashita, J BIOMED MATER RES B, 2010, 92, 120-128.

[18] N. Sahiner, W. Godbey, G.L. McPherson, V.T. John, Microgel, Colloid Polym Sci, 2006, 284, 11211129.

[19] C. Dalwadi, G. Patel, Recent Pat Nanotechnol, 2015, 9, 17-25.

[20] E. Hachet, H. Van den Berghe, E. Bayma, M.R. Block, R. Auzély-Velty, , Biomacromolecules, 2012, 13, 18181827.

[21] K.P. Krafts, Organogenesis, 2010 , 6, 225-233.

[22] M.W. Tibbitt, K.S. Anseth, Biotechnol Bioeng, 2009, 103, 655-663.

[23] T. Tokatlian, C. Cam, S.N. Siegman, Y. Lei, T. Segura, Acta biomaterialia, 2012, 8, 3921-3931.

[24] M. Hounslow, R. Ryall, V. Marshall, AIChE J, 1988, 34, 18211832.

[25] N. Sahiner, J. Xinqiao, Turk $J$ Chem, 2008, 32, 397-409.

[26] M. De Loos, B.L. Feringa, J.H. van Esch, Eur J Org Chem, 2005, 17, 3615-3631.

[27] M.N. Collins, C. Birkinshaw, J Appl Polym Sci, 2011, 120, 1040-1049. [28] Z.Q. Cao, G.J. Wang, Chem. Rec., 2016, 16, 1398-1435.

[29] X. Jia, K.L. Kiick, Macromol Biosci 2009, 9, 140-156. 
[30] J.K. Oh, R. Drumright, D.J. Siegwart, K. Matyjaszewski, Prog Polym Sci, 2008, 33, 448-477.

[31] J.K. Beattie, Lab Chip, 2006, 6, 1409-1411.

[32] J.D. Clogston, A.K. Patri, Springer, 2011, 697, 63-70.

[33] S.M. Dizaj, F. Lotfipour, M. Barzegar-Jalali, M.-H. Zarrintan, K. Adibkia, J DRUG DELIV SCI TEC, 2016, 35, 16-23.

[34] P.R. Mishra, L. Al Shaal, R.H. Müller, C.M. Keck, Int J Pharm, 2009, 371, 182-189.

[35] L. Wu, J. Zhang, W. Watanabe, Adv Drug Deliv Rev, 2011, 63, 456469.
[36] S. Honary, F. Zahir, J Pharm Res, 2013, 12, 265-273.

[37] S. Honary, M. Jahanshahi, P. Golbayani, P. Ebrahimi, K. Ghajar, $J$ Nanosci Nanotechnol, 2010, 10, 77527757.

[38] S. Sakulwech, N. Lourith, U. Ruktanonchai, M. Kanlayavattanakul, Asian J. Pharm. Sci., 2018, 13, 498504.

[39] C.E. Schanté, G. Zuber, C. Herlin, T.F. Vandamme, Carbohydr Polym, 2011, 85, 469-489.

[40] M. Al-Sibani, A. Al-Harrasi, R.H. Neubert, J Biochem Anal Stud, 2018, 3, 1-8.

[41] K. Tomihata, Y. Ikada, Biomaterials, 1997, 18, 189-195.

How to cite this manuscript: Elham Ahmadian, Solmaz Maleki Dizaj, Simin Sharifi, Aziz Eftekhari, Mohammad Samiei. Hyaluronic acid hydrogel nanoscaffolds: production and assessment of the physicochemical properties. Eurasian Chemical Communications, 2020, 2(1), 51-58. 